\title{
FROM FRAGMENTATION TO UNITY, FROM MODEL TO RESEARCH. THE CHALLENGES OF BUSINESS COMMUNICATION
}

\author{
by Edoardo Teodoro Brioschi
}

\section{Introduction}

The growing of communication importance and complexity dates back in the United States to the Seventies, and was specifically produced by a series of phenomena - inside and outside businesses themselves - of the greatest importance: amongst them, as it is frequently underlined, the development of the media of communication, as well as the wealth of data available regarding consumers and their behaviour, specifically as a result of the evolution of technology.

The same phenomena have undoubtedly had a similar effect, with the usual time lag and with some further difficulties in Europe, where nevertheless the potential for business communication, or better its requirements, changed as a result of even more general phenomena, some of which date back to a slightly earlier period.

We are considering specifically the ' 68 protest movements, which began in the United States and then spread to Europe, promoting a deep rethinking of the economy and even the society of Western countries, by contesting the concepts and values on which they had been traditionally founded.

Being an expression of a general renewal necessity, these protest movements - starting from the knowledge-based institutions - actually intended to conquer the whole society in order to proceed to its global refounding.

Here we need only to mention the contested concepts of development in a given country and the centrality of businesses.

Until the birth of protest movements, a country's development was seen mainly in terms of economic development, which was substantially supposed to drive social development. These movements stood the relationship on its head, with society and its aims becoming the

Mercati e Competitività n. 3, 2012 


\section{E.T. Brioschi}

foundation for a country's development, and economic models of development being realized only within these aims.

As for the centrality of businesses (essentially part of the centrality of economic development), their overriding importance in a country's development was tout court belittled. They have since struggled - in particular in the Eighties - to regain this role, but leveraging on their social responsibilities.

\section{The birth of total business communication}

The extreme relevance acquired by communication from the Seventies stems, in our opinion, precisely from the need felt by businesses, following the events of ' 68 , to make their objectives, products and policies more widely and promptly known, as well as to publicize initiatives that revealed their participation in the social (and not only economic) development of a country - or several countries in some cases - therefore enabling them to acquire that 'right of citizenship' that has been so widely discussed.

In this social and economic environment, the concept of total business communication has arisen.

The term business communication, as it was observed, intends expressing the connection and profound sharing, observable today perhaps more than in the past, between the business world and the communication world. In fact, there is a great interest - as it was also remarked on the businesses side - in the ability of the entrepreneur, carrying out business, to use communication as a resource and operational strategy for economic growth and, conversely, in communication to acquire economic consistence and to avoid the temptation to remain, on the surface, as a mere paint glistening on the company.

The concept of total business communication, whose introduction in Italy dates back to a contribution of ours in the early 1970s, holds that there is no element, no aspect, no activity of the business that is not reflected on the plane of communication and consequently all these factors (from the corporate name to the legal form, from the personnel to the facilities, from the whole set of policies to the positions in the outlet and supply markets and so forth) influence the identity and, through it, the image of the business itself.

Hence the need to govern, in a purposeful and coherent way, the complete set of elements, aspects and activities mentioned above, by implementing the general policy of governance deriving from a communication approach (total business communication policy or strategy). 


\section{From fragmentation to unity in business communication}

More precisely, the term total communication is meant to refer to the universe of events, through which the company activates a process of communication with one or more publics (individuals, companies or institutions), to which it proposes its own identity or one or more aspects of itself.

As for the events enhancing the communication process in question, they cover an exceptionally broad spectrum of contents, articulations and functionally involved periods of time.

These events can be divided into two classes with regard to the communicative value - implicit or explicit. Thus there are:

- some events that are explicitly developed by the company in order to activate one or more specific communication processes referring, therefore, to one or more publics;

- some implicit events related to the structure and organization of the company, having however in themselves a potential communicative value, that finds its own realization and reflection by means of the various publics (individuals, companies or institutions) with which the company enters into contact.

In any case, the theoretical premise underlying total business communication does not stem from a realization of the multiplicity of publics or activities and forms of communication, rendered available specifically by the development of technology, or from the explicit or implicit character of these activities or forms; but from the unity of the 'business' as an organization, as taught by Gino Zappa, the master of the modern Italian school of business economics.

Any business (whether private or public; industrial-, commercial- or service-based; local or international; real or virtual), considered in a holistic perspective, is a combination of factors and elements directed towards the satisfaction of specific human needs, whose total value should surpass that of the individual factors and elements in question, and which tends to operate generally in a medium to long term perspective, using adequate parameters of evaluation for that purpose.

But if we accept that the business is a unified organization and any initiative it undertakes acquires a precise significance only in the light of the business as a whole, the specific conditions in which this business operates and more generally the context, then - in a rational vision - even this specific factor called 'communication' cannot and should not be managed in a fragmentary way, arbitrarily separating activity from activity and instrument from instrument, but it should be addressed with a unified approach (though with full awareness of the multiple relationships with all the other 


\section{E.T. Brioschi}

factors and characteristics of the firm), hence giving the business an optimal contribution in that this contribution should be superior to the set of the results that would be attained by managing the individual communication activities separately.

\section{The communicative business}

Certainly the Italian school of thought fully realizes:

- the multiplicity of publics with which the business has to establish enduring relationships through adequate communication processes;

- the multiplicity of activities and instruments of communication (conventional and unconventional) that need to be used in appropriate combinations to activate, maintain and develop these relationships;

- the multiplicity of contents to be defined case by case, to achieve synergy by using them together and with the instruments/means chosen to disseminate them.

Thus what a multiplicity of publics, activities and contents that reflect the multiplicity of the objectives that the business - through communication or principally through it, or at any rate with its contribution - intends to achieve.

This, however, is not - as already remembered - the theoretical starting point of the concept of total business communication: if anything it is evidence of the complexity of the communication that the business should develop. A complexity that grows yet further if we consider that - as it was stated before - there is no element, aspect or activity of the business that does not make its contribution on the plane of communication, both on the level of the identity, and - through this - on the level of the image, or, as is also said today, the reputation of the business.

Essentially the business communicates with all of itself (total business communication), explicitly and implicitly, in ways which the management is fully aware of (communicative business) and also, unfortunately, in ways of which it is unaware or not sufficiently aware.

And, with reference to the awareness and the purposefulness of the management - and specifically the top management - in governing the communication of the business in all its aspects, there arises the basic problem of the existence or absence, in a specific business, of a culture of communication.

More exactly, the communicative business represents that particular business which - having developed a culture of communication - is characterized by the full acceptance of the communication approach, as well as the related policy or strategy that results. 
The communicative business is therefore a particular business model in line with the demands of the present environment and whose behaviour is exemplary for any company that thinks to apply the communication approach.

In this respect, it seems however fundamental to call to mind that, just like the business, its audiences, beginning with the consumers, are also making use of every media and vehicle at their disposal (as well as every source of information) to communicate - or counter-communicate - with the business as well as with every other person or organization.

In such a way a true problem of competition, specifically with the business, arises in the field of communication (business push communication against consumer pull communication), while the optimal goal should be without any doubt the one of cooperation between the two parties or perhaps, more realistically, the one of coopetition.

\section{From theory to implementation}

But, if the theoretical principles underpinning business communication appear to be quite clearly well developed, the question of why the application of these principles is still very limited even in the more highly developed countries naturally arises.

The problem is undoubtedly complex, given the need deriving from the so mentioned approach (communication approach) for a unified deployment - meaning one guided by an overall strategy - of the activities, means and forms of communication used by the business. There are first of all concrete difficulties that stand in the way of this requirement, just as there are advantages to be borne in mind.

The difficulties in question are specifically caused by:

- the disparity between the objectives which each area of business communication (institutional communication, internal communication, marketing communication and economic and financial communication) pursues, though they share a single final objective;

- the dispersal of the governance of these areas between different organizational functions, with the consequent difficulty of pursuing that final shared objective, because that objective ends up by reducing the power of the individual functions in terms of the responsibility for communication attributed to each;

- the increasing richness, complexity and specificity of the media and tools of communication, which may make it more difficult, even extremely difficult, to pursue the objective of a unified use, as posted by the theory; 


\section{E.T. Brioschi}

- the different degrees of importance attributed to the different areas of business communication in the sector where the business operates, in the individual business considered as a particular phase of its existence and, finally, in the business considered in its development.

Alongside the difficulties in question should be set the advantages of achieving the unified use of the activities, media, and forms of communication. This use is made indispensable by both the strategic role specific to business communication and the financial expenditure involved.

\section{From the model to innovative research}

What is then now, more specifically, the state-of-the-art in the business communication field?

During the past twenty years, in Italy we have carried out an extensive research activity on the evolution and governance of the various areas of communication.

This research experience has shown how companies require a huge effort (at different levels) to achieve an evolution of communication appropriate to present times and challenges:

- firstly at the company level, specifically at its culture of communication level, from which logically stems an attitude of top management that is of proper appreciation of the results of the communication approach and of its policies;

- then at the business communication theory level, a theory able to investigate - as part of a comprehensive and realistic plan, but with the progressivity which alone makes possible the implementation of such a plan - still unexplored or poorly explored areas of the potentialities of communication;

- finally, at the communication research level, so that research really applies and promotes theoretical development, abandoning outdated methods, and critically considering still used tools. Also, most importantly, experimenting with new methods and tools.

To be observed that, even if the research is called specifically to the third one of these levels, it is clear that it is able to influence both the theory and the culture of communication and, therefore, the attitude of top management. The research, in short, plays, within the evolutionary effort of business communication, a central role, when acting with determination within a theoretical and operational scheme of reference which is itself called to offer a specific and fundamental contribution. The mentioned in- 
stances of perfection and innovation in business communication research and practice do not concern only our country, but express an increasingly felt need at the international level.

But, more precisely, what may be the contribution of research to the model proposed by the concept of total business communication?

As a research topic, the model of total business communication is fundamentally in the descriptive stage. If the implementation of such concept gives rise to a communication system, involving the whole business, the task of the research will be logically the exploration of such system, beginning from the specific communication activities and their interrelations.

The last ones can be studied with reference to the two basic classes of publics (internal and external publics):

- before separately, taking into consideration everyone of the two communication sub-systems;

- after jointly, inside the business communication system originated from the two sub-systems.

In any case, the exploration must be extended also to the interrelations existing between the business communication system and the total system (grouping economic, technological, cultural and social variables) in which the business operates.

Following these methodological suggestions, it will be possible to strengthen the concept of total business communication and the related model in a progressive way.

This will have two consequences:

- one of general character, that is to enrich the concept of business itself and its unity, offering a new and meaningful perspective of analysis;

- one of specific character, that is to put pressure on the business governance in order to further rationalise the behaviour in the business communication field on local as well as international level. 